PROCEEDINGS OF THE AMERICAN MATHEMATICAL SOCIETY

Volume 124, Number 8, August 1996

\title{
A TOPOLOGICAL CHARACTERIZATION OF LINEARITY FOR QUASI-TRACES
}

\author{
L. J. BUNCE AND J. D. MAITLAND WRIGHT \\ (Communicated by Palle E. T. Jorgensen)
}

\begin{abstract}
Let $\mathcal{A}$ be a $C^{*}$-algebra, and let $\mu$ be a (local) quasi-trace on $\mathcal{A}$. Then $\mu$ is linear if, and only if, the restriction of $\mu$ to the closed unit ball of $\mathcal{A}$ is uniformly weakly continuous.
\end{abstract}

\section{INTRODUCTION}

Throughout this paper, $\mathcal{A}$ will be a $C^{*}$-algebra and $A$ will be the real Banach space of selfadjoint elements of $\mathcal{A}$. The closed unit ball of $\mathcal{A}$ is $\mathcal{A}_{1}$ and the closed unit ball of $A$ is $A_{1}$. We do not assume the existence of a unit in $\mathcal{A}$.

Definition. A quasi-linear functional on $\mathcal{A}$ is a function $\mu: \mathcal{A} \rightarrow \mathbb{C}$ with the following properties:

(1) $\mu$ is bounded on $\mathcal{A}_{1}$.

(2) Whenever $a$ and $b$ are in $A$ then

$$
\mu(a+i b)=\mu(a)+i \mu(b) .
$$

(3) Whenever $\mathcal{B}$ is an abelian $*$-subalgebra of $\mathcal{A}$ then the restriction of $\mu$ to $\mathcal{B}$ is linear.

Examples of non-linear, quasi-linear functionals on $M_{2}(\mathbb{C})$ exist in great abundance. See [7] for a full description of all the non-linear quasi-states on $M_{2}(\mathbb{C})$.

Definition. A quasi-linear functional, $\mu$, on $\mathcal{A}$ is said to be a quasi-trace if, whenever $x$ and $y$ are in $\mathcal{A}$,

$$
\mu(x y)=\mu(y x) .
$$

We do not require a quasi-trace to be positive on positive elements of $A$.

Handelman [12], Blackadar and Handelman [4] and Haagerup [11] have made powerful contributions to the problem of determining when quasi-traces are linear. They consider bi-traces, that is, positive quasi-traces which are postulated to be well-behaved with respect to tensoring with $M_{2}(\mathbb{C})$, the algebra of two-by-two complex matrices.

In this note, we impose no algebraic restrictions on the quasi-traces considered. Instead, we show that for a quasi-trace, linearity is equivalent to uniform weak continuity on the unit ball.

Received by the editors May 16, 1994 and, in revised form, January 24, 1995.

1991 Mathematics Subject Classification. Primary 46L30, 46L05.

(C)1996 American Mathematical Society 
Our proofs are brief because most of the hard work has been done elsewhere. In particular, we proved in [6] that:

Theorem. Let $\mathcal{A}$ be a $C^{*}$-algebra with no quotient isomorphic to $M_{2}(\mathbb{C})$. Let $\mu$ be a quasi-linear functional on $\mathcal{A}$. then $\mu$ is linear if, and only if, $\mu$ is uniformly weakly continuous on $\mathcal{A}_{1}$.

This result is best possible, in the sense that, if $\mathcal{A}$ does have a quotient onto $M_{2}(\mathbb{C})$, then there exists a quasi-linear functional $\mu$ on $\mathcal{A}$, where $\mu$ is uniformly weakly continuous on $\mathcal{A}$, and $\mu$ is not linear; see [8]. However, when $\mu$ is a quasitrace, we shall show that the analogue of the above theorem is true for all $\mathcal{A}$.

In fact, we shall use a weaker property than quasi-linearity, local quasi-linearity.

Definition. A function $\mu: \mathcal{A} \rightarrow \mathbb{C}$ is said to be locally quasi-linear if the following properties hold:

(1) $\mu$ is bounded on $\mathcal{A}_{1}$.

(2) Whenever $a$ and $b$ are in $A$ then

$$
\mu(a+i b)=\mu(a)+i \mu(b) .
$$

(3) Whenever $\mathcal{B}$ is a closed $*$-subalgebra of $\mathcal{A}$ generated by a single Hermitian element then the restriction of $\mu$ to $\mathcal{B}$ is linear.

It is clear that when $\mu$ is quasi-linear, then $\mu$ is locally quasi-linear. Surprisingly, the converse is false, even when $\mathcal{A}$ is abelian and separable. Aarnes [2] constructs a counter-example where $\mathcal{A}$ is the $C^{*}$-algebra of continuous functions on the unit square. However, whenever $\mathcal{A}$ is sufficiently rich in projections, in particular when $\mathcal{A}$ is a von Neumann algebra, then local quasi-linearity does imply quasi-linearity [3].

Definition. A function $\mu: \mathcal{A} \rightarrow \mathbb{C}$ is a local quasi-trace if $\mu$ is locally quasi-linear and, for each $x$ and $y$ in $\mathcal{A}$,

$$
\mu(x y)=\mu(y x)
$$

\section{TYPE $\mathrm{I}_{n}$ ALGEBRAS}

Let $S$ be a compact Hausdorff space, and let $C(S)$ be the commutative $C^{*}$ algebra of all continuous complex-valued functions on $S$. The selfadjoint part of $C(S)$ is a boundedly complete vector lattice if, and only if, $S$ is extremally disconnected, that is, the closure of each open subset of $S$ is also open. In all that follows we shall assume $S$ to be extremally disconnected.

Let $M_{n}(C(S))$ be the algebra of $n \times n$ matrices over $C(S)$. Then

$$
M_{n}(C(S))=C\left(S, M_{n}\right)=C(S) \otimes M_{n} .
$$

We equip $M_{n}(C(S))$ with the natural involution and $C^{*}$-norm. The canonical centre-valued trace, $\Gamma$, on $M_{n}(C(S))$ is defined by

$$
\Gamma\left[a_{i j}\right]=\frac{1}{n} \sum_{1}^{n} a_{r r} .
$$

Proposition 1.1. Let $\mu$ be a quasi-linear functional on $M_{n}(C(S))$ such that $\mu\left(U T U^{*}\right)=\mu T$ for each unitary $U$ and each selfadjoint $T$. Let $\bar{\mu}$ be defined on $C(S)$ by $\bar{\mu}(f)=\mu(f \otimes I)$. Then $\mu$ is linear and $\mu=\bar{\mu} \Gamma$. 
Proof. Let $\lambda=\bar{\mu} \Gamma-\mu$. Then $\lambda$ is quasi-linear and vanishes on the centre of $M_{n}(C(S))$. Moreover

$$
\lambda\left(U T U^{*}\right)=\lambda(T)
$$

for each unitary $U$ and selfadjoint $T$.

To simplify notation, we shall prove this proposition for $n=2$, which is what we shall need in the next section. The proof for general $n$ is a trivial extension of the argument.

For $x \in C(S)$ we observe that

$$
\left(\begin{array}{ll}
0 & 1 \\
1 & 0
\end{array}\right)\left(\begin{array}{ll}
x & 0 \\
0 & 0
\end{array}\right)\left(\begin{array}{ll}
0 & 1 \\
1 & 0
\end{array}\right)=\left(\begin{array}{ll}
0 & 0 \\
0 & x
\end{array}\right) .
$$

So

$$
\lambda\left(\begin{array}{ll}
x & 0 \\
0 & 0
\end{array}\right)=\lambda\left(\begin{array}{ll}
0 & 0 \\
0 & x
\end{array}\right)=\frac{1}{2} \lambda\left(\begin{array}{ll}
x & 0 \\
0 & x
\end{array}\right)=0 .
$$

Hence, for arbitrary $a$ and $b$ in $C(S)$,

$$
\lambda\left(\begin{array}{ll}
a & 0 \\
0 & b
\end{array}\right)=\lambda\left(\begin{array}{ll}
a & 0 \\
0 & 0
\end{array}\right)+\lambda\left(\begin{array}{ll}
0 & 0 \\
0 & b
\end{array}\right)=0 .
$$

Let $T$ be a selfadjoint element of $M_{2}(C(S))$. Then (see Deckard and Pearcy [10]), there exists a unitary $U$, such that

$$
U T U^{*}=\left(\begin{array}{ll}
a & 0 \\
0 & b
\end{array}\right)
$$

where $a$ and $b$ are in $C(S)$. The $\lambda(T)=\lambda\left(U T U^{*}\right)=0$. It follows that $\lambda$ vanishes on $M_{2}(C(S))$. So $\mu=\bar{\mu} \Gamma$ and hence $\mu$ is linear.

\section{LOCALLY QUASI-LINEAR TRACES}

Lemma 2.1. Let $\mathcal{M}$ be a von Neumann algebra, and let $\mu: \mathcal{M} \rightarrow \mathbb{C}$ be locally quasi-linear and such that $\mu\left(u x u^{*}\right)=\mu(x)$ for each unitary $u$ in $\mathcal{M}$ and each selfadjoint $x$ in $\mathcal{M}$. Then $\mu$ is linear.

Proof. Let $\mathcal{B}$ be any maximal abelian $*$-subalgebra of $\mathcal{M}$. Then $\mathcal{B}$ is generated by its projections and so, by [3], the locally quasi-linear $\mu$ is linear on $\mathcal{B}$. Thus $\mu$ is quasi-linear on $\mathcal{M}$.

Let $\mathcal{M}=e \mathcal{M} \oplus(1-e) \mathcal{M}$ where $e$ is a central projection such that $e \mathcal{M}$ is of Type $\mathrm{I}_{2}$ and $(1-e) \mathcal{M}$ has no direct summand of Type $\mathrm{I}_{2}$.

For any selfadjoint $x$ in $\mathcal{M}$, since $e$ is central,

$$
\mu(x)=\mu(e x)+\mu((1-e) x) .
$$

By Theorem B [5] or, in the case when $\mu \geq 0$ by Christensen [9] and Yeadon [12], the restriction of $\mu$ to $(1-e) \mathcal{M}$ is linear.

Since $e \mathcal{M}$ is of Type $\mathrm{I}_{2}$,

$$
e \mathcal{M} \approx M_{2}(C(S))
$$

where $S$ is extremally disconnected. (In fact, $S$ is hyperstonian.) So, by Proposition 1.1 , the restriction of $\mu$ to $e \mathcal{M}$ is linear. Hence $\mu$ is linear on $\mathcal{M}$. 
Theorem 2.2. Let $\mu: \mathcal{A} \rightarrow \mathbb{C}$ be locally quasi-linear. Suppose that either

(i) $\mu(x y)=\mu(y x)$ for all $x$ and all $y$ in $\mathcal{A}$ or

(ii) $\mathcal{A}$ has a unit and, for each unitary $u$ and selfadjoint $x, \mu\left(u x u^{*}\right)=\mu(x)$.

Then $\mu$ is linear if, and only if, $\mu$ is uniformly continuous on $A_{1}$ with respect to the weak topology.

Proof. By Lemma 1.1 [6], when $\mu$ is linear then $\mu$ is uniformly continuous on $\mathcal{A}_{1}$ with respect to the weak topology.

Let us now suppose that $\mu$ is uniformly weakly continuous on $\mathcal{A}_{1}$.

Let $H$ be the universal representation space of $\mathcal{A}$. We recall that $\mathcal{A}^{* *}$, the second dual of $\mathcal{A}$, may be identified with the von Neumann envelope of $\mathcal{A}$ in $\mathcal{L}(H)$.

Then, by the proof of Theorem $3.1[6], \mu$ extends uniquely to a locally quasilinear functional $\bar{\mu}: \mathcal{A}^{* *} \rightarrow \mathbb{C}$, where $\bar{\mu}$ is continuous on $\mathcal{A}^{* *}$ with respect to the strong operator topology of $\mathcal{L}(H)$.

We recall that $\mathcal{A}_{1}$ is strongly dense in the unit ball of $\mathcal{A}^{* *}$. Hence, if (i) is satisfied, whenever $x$ and $y$ are in $\mathcal{A}^{* *}$, there exist bounded nets in $\mathcal{A},\left(x_{i}\right)$ and $\left(y_{j}\right)$, where $x_{i} \rightarrow x$ and $y_{j} \rightarrow y$ is the strong operator topology of $\mathcal{L}(H)$.

$$
\bar{\mu}\left(x_{i} y_{j}\right)=\mu\left(x_{i} y_{j}\right)=\mu\left(y_{j} x_{i}\right)=\bar{\mu}\left(y_{j} x_{i}\right) .
$$

So

$$
\bar{\mu}\left(x y_{j}\right)=\bar{\mu}\left(y_{j} x\right) .
$$

Hence

$$
\bar{\mu}(x y)=\bar{\mu}(y x) .
$$

Alternatively, if (ii) is satisfied, then $1 \in \mathcal{A}$ and, for each unitary $u$ in $\mathcal{A}^{* *}$, there is a net of unitaries $\left(u_{j}\right)$ in $\mathcal{A}$ which converges strongly to $u$ in $\mathcal{L}(H)$. Hence $\bar{\mu}\left(u x u^{*}\right)=\bar{\mu}(x)$ for each selfadjoint $x$ in $\mathcal{A}$. Hence $\bar{\mu}\left(u x u^{*}\right)=\bar{\mu}(x)$ for each selfadjoint $x$ in $\mathcal{A} * * *$.

We may now apply Lemma 2.1 to deduce that $\bar{\mu}$ is linear on $\mathcal{A}^{* *}$ and hence $\mu$ is linear on $\mathcal{A}$.

\section{REFERENCES}

1. J. F. Aarnes, Quasi-states on a $C^{*}$-algebra, Trans. Amer. Math. Soc. 14 (1970), 601-625. MR 43:8311

2.

3. C. A. Akemann and S. M. Newberger, Physical states on $C^{*}$-algebras, Proc. Amer. Math. Soc 40 (1973), 500. MR 47:7406

4. B. Blackadar and D. Handelman, Dimension functions and traces on $C^{*}$-algebras, J. Funct. Anal. 45 (1982), 297-340. MR 83g:46050

5. L. J. Bunce and J. D. M. Wright, The Mackey-Gleason problem for vector measures on projections in von Newmann algebras, J. London Math. Soc 49 (1994), 133-149. MR 95b:46090

6. - The quasi-linearity problem for $C^{*}$-algebras Pacific J. Math. 12 (1994), 271-280. MR 95h:46102

7. Skew-symmetric functions on the sphere and quantum measures, Expositiones Math. 12 (1994), 271-280. MR 95h:46102

8. _ Non-linearity and approximate additivity, Expositiones Math. 12 (1994), 363-370. CMP 95:02.

9. E. Christensen, Measures on projections and physical states, Comm. Math. Phys. 86 (1982), 529-538. MR 85b:46072

10. D. Deckard and C. Pearcy, On matrices over the ring of continuous complex valued functions on a Stonian space, Proc. Amer. Math. Soc. 14 (1963), 322-328. MR 26:5438 
11. U. Haagerup, Quasitraces on exact $C^{*}$-algebras, manuscript.

12. D. Handelman, Homomorphisms of $C^{*}$-algebras to finite $A W^{*}$-algebras, Michigan Math. J. 28 1981, 229-240. MR 82h:46078

13. F. W. Yeadon, Finitely additive measures on projections in finite $W^{*}$-algebras, Bull. London Math. Soc. 16 1984, 145-150. MR 85i:46087

Department of Mathematics, The University of Reading, White Knights, P. O. Box 220, Reading RG6 2AX, England

Isaac Newton Institute, 20 Clarkson Road, Cambridge, England 